\title{
Die Übernahme von Budget- verantwortung durch Anbieter der Integrierten Versorgung
}

Die Übernahme von Budgetverantwortung durch die Anbieter neuer Versorgungsformen gewinnt mit der zunehmenden Einführung von populationsbezogenen Verträgen an Bedeutung. Selbst wenn eine derartige Zielvorgabe in entsprechenden Projekten bisher häufig keine explizite Relevanz für die Vergütung besitzt, wird eine Budgetbestimmung zumindest als Vorrausetzung für eine ökonomische Erfolgsmessung erforderlich. Im Folgenden sollen daher grundlegende Gestaltungsmöglichkeiten für eine Budgetverantwortung erläutert werden. Damit liefern die Ausführungen einen Überblick über die denkbaren Optionen und sensibilisieren für die möglichen Fallstricke in der praktischen Umsetzung.

\section{Dipl.-Ök. Sebastian Braun, Prof. Dr. Wolfgang Greiner}

\section{Sozialgesetzlicher Hintergrund}

Zum 1. April 2007 ist das Gesetz zur Stärkung des Wettbewerbs in der Gesetzlichen Krankenversicherung oder kurz GKV-Wettbewerbsstärkungsgesetz (GKV-WSG) in Kraft getreten. Zusammen mit dem Vertragsarztrechtsänderungsgesetz (VÄndG), welches am 1. Februar 2007 in Kraft trat, wurde das Gesundheitssystem in wichtigen Punkten verändert.

Auch wenn die Änderungen für die Integrierte Versorgung eher gering waren, wird die weitere Entwicklung stark von den Änderungen in anderen Bereichen des SGB $\mathrm{V}$ beeinflusst. Zudem wird die Anschubfinanzierung mit größter Wahrscheinlichkeit nicht über das Jahr 2008 hinaus verlängert.

Laut GKV-WSG sollen die Verträge zur Integrationsversorgung zukünftig eine bevölkerungsbezogene Flächendeckung der Versorgung ermöglichen. ${ }^{1}$ Neben den direkt die Integrierte Versorgung betreffenden gesetzlichen Neuerungen haben insbesondere der Gesundheitsfonds ${ }^{2}$, die

Dipl.-Ök. Sebastian Braun, Universität Hannover, Fakultät für Wirtschaftswissenschaften, Forschungsstelle für Gesundheitsökonomie

Prof. Dr. Wolfgang Greiner, Universität Bielefeld, Fakultät für Gesundheitswissenschaften, Lehrstuhl für Gesundheitsökonomie und Gesundheitsmanagement
Wahltarife ${ }^{3}$ und die Neuregelung der ambulanten Vergütung ${ }^{4}$ einen starken Einfluss auf die zukünftigen Entwicklungsperspektiven.

Ab dem 01.01.2009 soll der Gesundheitsfonds als Kernstück der Gesundheitsreform 2007 eingeführt werden. In diesem Zusammenhang wird die Bundesregierung erstmals Ende 2008 einen einheitlichen Beitragssatz festlegen. Den einzelnen Kassen bleibt nur die Möglichkeit, für ihre Mitglieder Zusatzbeiträge festzusetzen bzw. Erstattungen vorzunehmen. In Verbindung mit der Einführung von Wahltarifen innerhalb der gesetzlichen Krankenversicherung durch die Änderung von § 53 des SGB V ergeben sich verstärkte Anreize zu selektiven Verträgen.

Die Tarife gliedern sich auf in solche, die von den Krankenkassen angeboten werden müssen und die, deren Einführung für die Krankenversicherungen freiwillig ist. Sofern die Krankenkassen besondere Behandlungsprogramme anbieten, müssen sie seit 01.04.2007 Tarife für die Integrierte Versorgung, die besondere ambulante Versorgung, strukturierte Behandlungsprogramme für chronisch Kranke und eine hausarztzentrierte Versorgung anbieten. ${ }^{5}$ Optional können ebenfalls seit dem 01.04.2007 Selbstbehalttarife, Tarife für Nichtinanspruchnahme von Leistungen, variable Kostenerstattungstarife und Tarife zur Übernahme der über die Regelversorgung hinausgehende Kosten für Arzneimittel einer besonderen Therapierich- 


\section{THEMA}

tung angeboten werden. Grundsätzlich ist der Abschluss solcher Wahltarife für die Versicherten freiwillig.

Ein strategischer Ansatz aus Sicht der Krankenkassen ist es daher, ihr Engagement im Bereich der neuen Versorgungsformen vor dem Start des Fonds auszubauen, um ihren teilnehmenden Versicherten beispielsweise Tarife anzubieten, die günstiger sind als der festgelegte Einheitsbeitrag. Gerade eine populationsorientierte Vollversorgung der Versicherten eignet sich hier besonders.

Einen weiteren wichtigen Einflussfaktor stellt die Reform der ambulanten ärztlichen Vergütung dar. Bisher ergeben sich die Ausgaben als die Summe der historisch gewachsenen, kassenbezogenen Kopfpauschalen pro Mitglied. In Zukunft soll das Morbiditätsrisiko auf die Krankenkassen zurückverlagert werden, indem ärztliche Leistungen zu festen Europreisen vergütet werden. ${ }^{6}$ Folglich ergeben sich für die Kassen ab dem Jahr 2009 zusätzlich zu der Umstellung der Finanzierung über den Gesundheitsfonds auch im ambulanten Sektor erhebliche finanzielle Risiken. Betroffen werden insbesondere Kassen mit bisher vergleichsweise niedrigeren Kopfpauschalen sein.

\section{Mögliche Konsequenzen für die Integrationsverträge}

Die jetzige Situation, in der den Trägern der Integrierten Versorgung häufig alle zusätzlich angebotenen Leistungen extra vergütet werden und die sonstige Abrechnung wie in der Regelversorgung läuft, wird gegenüber den beteiligten Krankenkassen vor dem Hintergrund der zuvor erläuterten gesetzlichen Entwicklungen nicht einfach durchzusetzen sein. Die gesetzlichen Änderungen setzen stattdessen verstärkte Anreize, die Budgetverantwortung für den gesamten ambulanten Bereich und gegebenenfalls für die veranlassten Leistungen ab 2009 zu übergeben.

Folgende strategischen Überlegungen spielen dabei aus Sicht der Krankenkasse eine zentrale Rolle:

- Auch kurzfristig keine zusätzliche finanzielle Belastung

- Wettbewerb mit anderen Kassen um einen günstigeren Tarif (Wahltarife, Gesundheitsfonds)

- Vermeidung des Kostenrisikos im Zusammenhang mit der Umstellung der ambulanten Vergütung

- Teilweise Übertragung des Morbiditätsrisikos auf die beteiligten Leistungserbringer

- Sicherung der Versorgungsqualität und freiwilliger Präventionsanreiz für die Leistungserbringer (ganzheitliche Versorgung aus einer Hand)

Daraus ergeben sich zunächst erhebliche Interessenkonflikte zwischen Anbieter der Integrierten Versorgung und Krankenkassen. Auf Leistungserbringerseite besteht jedoch die Möglichkeit sich bei zunehmender Wettbewerbsintensität für die Zukunft zu positionieren und ihren Patienten eine hochwertige Versorgung abseits der Regelversorgung anzubieten. Außerdem können auch weiterhin zusätzliche
Einnahmen unabhängig von der Regelversorgung generiert werden.

\section{Ausgestaltungsprinzipien für eine Budgetverantwortung}

Die Übernahme von Budgetverantwortung durch einen Träger der Integrierten Versorgung erfordert die Konkretisierung folgender Punkte ${ }^{7}$ :

Umfang der Budgetverantwortung

Kalkulation eines Budgets als Sollgröße

Konsequenzen aus Budgetüberschuss bzw. -defizit

Laufzeit bzw. Gültigkeit der Budgetvereinbarung

\section{Umfang der Budgetverantwortung}

Hier ist zunächst zu klären, für welche Versichertenpopulation der Träger die Verantwortung übernehmen soll. Denkbar wären hier zum einen alle eingeschrieben Versicherten, zum anderen alle potentiellen Teilnehmer gemäß definierter Postleitzahlen oder Regionsgrenzen.

Beide Möglichkeiten haben spezifische Vor- und Nachteile. Eine Begrenzung auf die eingeschriebenen Versicherten stellt einen klaren Versichertenbezug her. Durch die Einschreibung und möglicherweise abgegebene Selbstverpflichtung des Patienten können Verhaltensregeln, wie z. B. die ausschließliche Behandlung durch am Vertrag teilnehmende Leistungserbringer und ausgewählte Kooperationspartner, gefestigt werden. Eine Ausweitung der Verantwortung auf alle zur Einschreibung berechtigten Versicherten hat dagegen den Vorteil, dass die Risikostruktur von Teilnehmern und Nichtteilnehmern unerheblich wird und dass das Budget nicht mit der Einschreibquote variiert.

Anschließend sollten die einzubeziehenden Leistungssektoren definiert werden. Theoretisch besteht insbesondere bei budgetbasierten Vergütungssystemen ein Anreiz für die Leistungserbringer, Leistungen auf andere Sektoren $\mathrm{zu}$ verschieben, $\mathrm{um}$ das eigene Budget $\mathrm{zu}$ schonen. Aus diesem Grund sollten möglichst alle Leistungsbereiche, auf die die beteiligten Leistungserbringer Einfluss nehmen können, einbezogen werden. Auch der Gesetzgeber geht von einer Übernahme der Budgetverantwortung insgesamt oder für definierte Teilbereiche (Kombiniertes Budget) aus. ${ }^{8}$

Aus Sicht der Leistungsrebringer erhöht sich durch den Einbezug mehrerer Sektoren der Steuerungsspielraum erheblich und es können verstärkt Integrationseffekte mit entsprechenden Einsparungen realisiert werden.

Als letztes wird der Umfang der Budgetverantwortung gegebenenfalls durch die Absicherung von Hochkostenfällen bestimmt. Das Budget kann insbesondere bei vergleichsweise geringen Teilnehmerzahlen sehr stark durch einzelne Hochkostenfälle belastet werden. Aber auch für größere 
Populationen ist eine entsprechende Vereinbarung aus mehreren Gründen sinnvoll.

Durch eine pauschale Budgetabgeltung sowohl für etwaige Präventionsleistungen als auch die Leistungen für Behandlungen, die durch die Leistungserbringer nicht oder nur gering beeinflusst werden können, überträgt man ökonomisch und versicherungstechnisch betrachtet einen Teil der Versicherungsfunktion auf einen dafür zumindest grundsätzlich nicht vorgesehenen Akteur. Ziel ist es ja gerade, den Leistungserbringern Anreize zu geben, ihren Steuerungsspielraum optimal zu nutzen und sie nicht durch unvermeidbare Krankheitskosten zu belasten. Außerdem hätten die Krankenkassen im Falle einer unzureichenden Absicherung gegen Hochkostenfällen gegebenenfalls eine entsprechend hohe Risikoprämie an die Leistungserbringer zu zahlen.

Ein weiterer Grund besteht in der Verminderung des Anreizes zur Selektion von Hochkostenrisiken durch die Vertragspartner. Gerade im Falle von Einschreibemodellen kann es diesbezüglich zu unerwünschten Effekten kommen, sofern der wirtschaftliche Druck auf die Leistungserbringer zu stark ist.

Theoretisch kann das Hochkostenrisiko ausschließlich von den Leistungserbringern, von den beteiligten Krankenkassen oder einem externen Rückversicherer getragen werden. Erstere Möglichkeit kommt nur bei einem entsprechend großen Risikopool und gleichzeitig einem in der Höhe streng limitierten Kostenrisiko in Frage. Bei einer sehr großen Teilnehmerzahl wäre beispielsweise eine Begrenzung auf den ambulanten Sektor eine Risikolimitierung.

Im Falle der Risikoübernahme durch die Krankenkasse oder einen externen Rückversicherer sollte ein Teil des Risikos beim Leistungserbringer bleiben, um den Anreiz zum wirtschaftlichen Handeln nicht zu mindern. (Risksharing)

Im Vorfeld müssen geeignete Auslöser definiert werden, bei denen die Absicherung durch die Kassen oder Rückversicherer greift. Denkbar ist hier ein Grenzbetrag pro Teilnehmer für einzelne oder alle einbezogenen Sektoren oder die Diagnose festgelegter Erkrankungen bzw. die Einschreibung in ein DMP-Programm.

Sind geeignete Auslöser gefunden, muss nur noch die geeignete Risikoteilung bzw. der von den jeweiligen Vertragspartnern zu verantwortende Anteil der Kosten operationalisiert werden. Sinnvoll kann hier eine prozentuale Beteiligung der Leistungserbringer entweder an den Gesamtkosten der betroffenen Versicherten oder im Falle eines Grenzbetrages an den darüber hinausgehenden Kosten sein.

\section{Budgetkalkulationsmethoden}

Grundsätzlich gibt es die Möglichkeiten, ein Budget entweder anhand der Vorjahreskosten der teilnehmenden Versicherten oder mit Hilfe einer Kontrollgruppe zu kalkulieren.
Die letztgenannte Methodik scheitert meist am Vorhandensein einer realen Kontrollgruppe. In diesem Fall kann auf Referenzwerte in Form von standardisierten Leistungsausgaben zurückgegriffen werden. Auch hier muss aber eine Einigung gefunden werden, welche Referenzwerte zur Anwendung kommen. Es ist abzuwägen, ob eigene Werte ermittelt werden oder ob vorhandene Quellen, beispielsweise die im Rahmen des Risikostrukturausgleichs berechneten Normkosten, genutzt werden. Wichtigstes Entscheidungskriterium ist, dass die regionalen und kassenspezifischen Besonderheiten in adäquater Weise Berücksichtigung finden.

Die Ausgaben des Vorjahres bzw. der letzten Abrechnungsperiode lassen sich verhältnismäßig einfach anhand von pseudonymisierten Daten der beteiligten Krankenkassen ermitteln. Ein Problem stellen allerdings die ambulanten Kosten dar.

Eine Möglichkeit besteht darin, die abgerechneten EBM-Punkte mit einem kalkulatorischen Punktwert zu bewerten. In diesem Fall stellt sich die Frage, welcher Wert verwendet werden soll. Dem seit 1. April 2005 gültigen EBM lag ein kalkulatorischer Punktwert von 5,11 Cent ${ }^{9}$ zu Grunde. Tatsächlich liegt der Punktwert jedoch in der Regel darunter.

Aus Sicht der Krankenkassen sind die realen Kosten begrenzt. Sie zahlen momentan eine kassenindividuelle Kopfpauschale je Mitglied. Diese ist völlig unabhängig von den abgerechneten Leistungen. Daher besteht die zweite Möglichkeit darin, die Summe über die gezahlten Kopfpauschalen zu bilden. Dabei ist zu beachten, dass für Familienversicherte keine Kopfpauschale gezahlt wird. Die Anzahl von Versicherten mit diesem Status muss also zuvor herausgerechnet werden.

Die erhobenen Kosten stellen eine wichtige Kennzahl für die Festlegung eines Budgets als Sollgröße dar. Unter Berücksichtigung des zuvor festgelegten Budgetumfanges sind bei dieser Kalkulationsmethode folgende Varianten denkbar:

- Gesamtausgaben des Vorjahres

- Gesamtausgaben des Vorjahres unter Berücksichtigung des Umgangs mit Hochkostenfällen

- Gesamtausgaben des Vorjahres unter Berücksichtigung eines allgemeinen Steigerungssatzes der Kassenausgaben

Insbesondere die letzten Varianten können auch kombiniert eingesetzt werden. Der Steigerungssatz kann anhand der allgemeinen GKV-Daten oder aber den regionalen, kassenspezifischen Ausgaben ermittelt werden. Dabei ist insbesondere eine differenzierte Betrachtung der jeweils einbezogenen Leistungsbereiche zu berücksichtigen. Je nach gesetzlicher Regulierung werden sich beispielsweise die Ausgaben für Arzneimittel und die ambulante Versorgung sehr unterschiedlich entwickeln. 


\section{Konsequenzen aus Budgetüberschuss bzw. -defizit}

Das so ermittelte Budget kann nach Ablauf eines festgelegten Zeitraums mit den tatsächlichen Kosten verglichen werden. Theoretisch kann dies auch nur durch das interne Vertragsmanagement geschehen, falls bezüglich der Konsequenzen festgelegt wurde, dass Gewinne oder Verluste allein die Leistungserbringer betreffen.

Mögliche andere Konsequenzen wären:

Der Budgetüberschuss bleibt bei den Leistungserbringern. Im Falle von Verlusten übernimmt die Krankenkasse entweder ganz oder teilweise.

- Krankenkassen und Leistungserbringer teilen sich die Gewinne oder Verluste gemäß einem vorher festgelegten Anteil.

Diese Entscheidung hängt allerdings sehr stark von der Ausgestaltung des zuvor festgelegten Budgets ab. Ist hier beispielsweise das Verlustrisiko durch eine Absicherung von Hochkostenfällen deutlich begrenzt, so ist das alleinige Tragen der Konsequenzen durch den Träger der Integrierten Versorgung möglicherweise zumutbar.

\section{Laufzeit der Budgetvereinbarung}

Einen wichtigen Schritt in den Vertragsverhandlungen zur Übernahme von Budgetverantwortung durch Leistungserbringer stellt die Festlegung der Laufzeit bzw. Gültigkeit des kalkulierten Budgets dar.

Angesichts des hohen Implementationsaufwandes, ist eine kurze Laufzeit nicht zu rechtfertigen. Außerdem ist eine langfristige Planungssicherheit aus Sicht beider Vertragsparteinen wünschenswert. Für die Krankenkassen lohnen sich in diesem Fall zum Beispiel entsprechende Anfangsinvestitionen oder die Auflegung eines speziellen Wahltarifes. Aus Sicht der Leistungserbringer ist es im Gegenzug ökonomisch rational, die Teilnehmer durch einen hohen präventiven Leistungsanteil dauerhaft gesund zu erhalten. Dennoch können vorzeitige Ausstiegsoptionen vereinbart werden.

Eine lange Laufzeit bedeutet aber nicht, dass die für das erste Jahr kalkulierte Budgethöhe bis zum Ende unverändert bleibt. Theoretisch kann das Budget jedes Jahr neu kalkuliert werden. Dies hat jedoch neben dem organisatorischen Aufwand den Nachteil, dass die Leistungserbringer für Kosteneinsparungen in den Folgeperioden bestraft werden, indem sie ein immer knapperes Budget erhalten.

Sinnvoll scheint daher stattdessen eine Einigung auf eine jährliche Anpassung des erstmals festgelegten Budgets um einen allgemeinen Steigerungssatz der regionalen Kassenausgaben.

\section{Abschließende Beurteilung}

In Anbetracht des aktuellen sozialpolitischen Hintergrunds sollten die Vertragspartner einer Integrierten
Versorgung eine mögliche Budgetverantwortung diskutieren. Die vorherigen theoretischen Überlegungen können einen ersten Orientierungspunkt für die weiteren Verhandlungen zwischen den beteiligten Parteien liefern.

Die Weiterentwicklung hin zu einem hoch innovativen Versorgungsmodell abseits der Vergütungsstrukturen der Regelversorgung bietet für alle Beteiligten große Chancen. Wichtigster Erfolgsfaktor ist die geeignete Budgetkalkulation.

Die aus gesundheitsökonomischer Sicht bei den weiteren Verhandlungen zu beachtenden Parameter wurden im Rahmen dieses Artikels erläutert. Außerdem konnten die Ausführungen erste Anhaltspunkte für die praktische Umsetzbarkeit liefern.

Da es bisher im deutschen Gesundheitswesen kaum Erfahrungen zu den tatsächlichen Auswirkungen von Budgetverantwortungsmodellen gibt, können sich gerade in den Anfangsjahren Defizite in der Budgetkalkulation zeigen. Eine begleitende Evaluation ist unerlässlich, um Schwachstellen aufzudecken und entsprechende Anpassungen zu initiieren.

\section{Literaturverzeichnis}

- Fünftes Buch Sozialgesetzbuch - Gesetzliche Krankenversicherung (2007):

Artikel 1 des Gesetzes vom 20. Dezember 1988, BGBl. I S. 2477, zuletzt geändert durch $\S 22$ Abs. 7 des Gesetzes vom 12. Dezember 2007 (BGB1. I S. 2861 Änderung durch Art. 5 G v. 19.12.2007 I 3024 (Nr. 67) noch nicht berücksichtigt)

- Gesetz zur Änderung des Vertragsarztrechts und anderer Gesetze (2006):

Vom 22. Dezember 2006 (BGBl. I S. 3439) ausgegeben zu Bonn am 30. Dezember 2006.

- Gesetz zur Stärkung des Wettbewerbs in der gesetzlichen Krankenversicherung (2007):

Vom 26. März 2007 (BGBl. I S. 378) ausgegeben zu Bonn am 30. März 2007.

- Kassenärztliche Bundesvereinigung (2004):

Beschluss des Bewertungsausschusses gem. §87 Abs. 3 SGB V in seiner 89. Sitzung am 13.05.2004, URL:http://daris.kbv.de/daris/doccontent dll?LibraryName $=$ EXTDARIS^DMSSLAVE\&SystemType $=2 \&$ LogonId $=$ ab0d6384710f89e675921e2bb911b2e6\&DocId=003745298\&Page $=1$ [Stand 20.05.2008]

- Lindenthal, J. / Sohn, S. / Schöffski, O. (2004): Praxisnetze der nächsten Generation: Ziele Mittelverteilung und Steuerungsmechanismen, Schriften zur Gesundheitsökonomie 3, Burgdorf.

\section{Fußnoten}

1 Vgl. § 140 a Abs. 1 Satz 2 SGB V.

2 Vgl. § 271 SGB V.

3 Vgl. § 53 SGB V.

4 Vgl. $§ 87$ a ff. SGB V.

5 Vgl. §53 Abs. 3 SGB V

6 Vgl. $\$ 87$ a Abs. 2 SGB V

7 Vgl. Lindenthal, J. / Sohn, S. / Schöffski, O. (2004), S. 22

8 Vgl. § 140 c Abs. 2 Satz 1 SGB V.

9 Vgl. Kassenärztliche Bundesvereinigung (2004), S. 11. 